\title{
Evaluación y seguimiento de planes de ordenamiento territorial en los municipios de Colombia ${ }^{1}$
}

\author{
Assessment and Monitoring of Land \\ Management Plans in the Municipalities of \\ Colombia
}

\section{Avaliação e Acompanhamento dos planos de ordenamento territorial nos municípios da Colômbia}

\author{
Ángela Natalia Camelo Garzón ${ }^{2}$ \\ Investigadora de la Universidad del Valle, Cali-Colombia \\ angela.camelo@correounivalle.edu.co \\ Leonardo Solarte-Pazos ${ }^{3}$ \\ Profesor e investigador de la Universidad del Valle, Cali-Colombia \\ leonardo.solarte@correounivalle.edu.co \\ Oswaldo López ${ }^{4}$ \\ Profesor e investigador de la Universidad del Valle, Cali-Colombia \\ oswaldo.lopez@correounivalle.edu.co
}

Recibido: 15.05 .14

Aprobado: 21.10 .14

1 Este artículo surge de un esfuerzo colaborativo entre académicos de diversas disciplinas en el marco de un proyecto de investigación de la Maestría de Administración de la Universidad del Valle, con el apoyo de las Maestrías de Políticas Públicas y de Arquitectura y Urbanismo. En él convergen tres áreas de conocimiento representadas por los grupos de investigación en Gestión y Evaluación de Programas y Proyectos de la Facultad de Ciencias de la Administración, y en Hábitat y desarrollo sostenible de la Escuela de Arquitectura de la Universidad del Valle.

2 Magíster en Administración.

3 Doctor en Administración. Maestría en Estudios políticos.

4 Doctor en Urbanismo. Magíster en Gestión ambiental urbana. 


\title{
Resumen
}

El proceso de ordenamiento territorial que se viene realizando en el país a partir de la expedición de la Ley 388 de 1997 (de Desarrollo territorial), es quizás una de las transformaciones más substanciales que ha sufrido el ejercicio de la planificación de los municipios en Colombia. Sin embargo, aún existe un gran camino por recorrer hacia la implementación de una verdadera cultura de planificación y de gestión integral del territorio. En este contexto, el presente artículo presenta los resultados de un proyecto de investigación que propone los fundamentos de un modelo de evaluación de los planes de ordenamiento territorial municipal en el país. Su particularidad radica en la integración de elementos tradicionales de la gestión urbana con un enfoque evaluativo de gestión pública y social.

Palabras clave: Modelo de Evaluación y Seguimiento, Planes de Ordenamiento Territorial.

\begin{abstract}
The Planning Process for land use that is being performed in Colombia according to Law 388, issued in 1997 (Land Development Law), is perhaps one of the most substantial changes it has undergone the exercise of planning by municipalities in Colombia. However, there is still a long way to go in order to implement a true culture of comprehensive planning and land management. In this context, the present article aims to show the results of a research project which proposes an alternative approach to monitoring and evaluating management of land use planning for municipalities in Colombia, in the specific context of city planning. This new approach integrates traditional urban management elements with public and social management assessment focus.
\end{abstract}

Keywords: Assessment and Monitoring Model, Land use Planning.

\section{Resumo}

O processo de ordenamento territorial que vem se realizando no país, após a promulgação da Lei 388 de 1997 (de Desenvolvimento Territorial), é talvez uma das transformações mais substanciais no exercício de planejamento dos municípios na Colômbia. No entanto, ainda há um longo caminho por percorrer perante a implementação de uma verdadeira cultura de planejamento e gestão integrada da terra. Neste contexto, o presente artigo apresenta os resultados de um projeto de pesquisa que propõe as bases de um modelo de avaliação dos Planos de Ordenamento Territorial. Sua singularidade reside na integração de elementos tradicionais de gestão urbana com uma abordagem avaliativa de gestão pública e social.

Palavras-chave: Modelo de Avaliação e Monitoramento, Planos de Ordenamento Territorial. 


\section{Introducción}

El proceso de ordenamiento territorial que se viene realizando en el país a partir de la expedición de la Ley 388 de 1997 (de Desarrollo territorial), es quizás una de las transformaciones más substanciales que ha sufrido el ejercicio de la planificación de los municipios en Colombia. Los Planes de Ordenamiento Territorial (POT) constituyen el instrumento más importante dentro de esta nueva forma de configurar el territorio y buscan, a partir de un diagnóstico físico y socioeconómico de los municipios y regiones, definir las directrices que guiarán el desarrollo físico durante su vigencia, orientados por una "visión de futuro deseado" construida de manera colectiva.

Los POT se adoptaron, a partir de las teorías de la administración y de la gestión pública, específicamente desde la "planeación estratégica", con el fin de garantizar que las acciones planificadas apuntaran al logro de objetivos de una manera eficiente e integrada a una visión de largo plazo (filosofía de gestión), mediano plazo y corto plazo.

Esta nueva orientación que se le dio al desarrollo territorial municipal y posteriormente regional, basada en modelos de planificación estratégica, requiere igualmente plantear estrategias y métodos que permitan hacer seguimiento a las actuaciones físicas y evaluar el impacto de dichos planes en los territorios y en su población, principalmente para vigilar si los resultados prácticos se conforman lo más exactamente posible a las metas propuestas y tomar los correctivos necesarios.

Este nivel de seguimiento y evaluación es mucho más complejo y estratégico para el desarrollo de las regiones, que el simple seguimiento y conocimiento individual de los proyectos involucrados en el plan. Para ello, la misma Ley 388 de 1997 dispuso que los municipios debían implementar un sistema de seguimiento y evaluación a la ejecución de los planes que garantice la integridad del proceso de ordenamiento, a la vez que proporcione sustento técnico para determinar los ajustes necesarios que se requieran para encaminarlo hacia la dinámica que esté tomando el desarrollo del territorio (artículos 24 y 112). Sin embargo, la gran mayoría de los municipios y distritos no lo hicieron, debido a que las administraciones priorizaron la elaboración y puesta en marcha de su POT y no aseguraron la provisión de recursos (técnicos y financieros) para desarrollar e implementar un sistema de evaluación y seguimiento.

Este artículo se focaliza en la necesidad de proveer a los gobiernos locales de herramientas que permitan monitorear y evaluar sus POT, cumpliendo con las exigencias de la ley, y asegurando información de carácter gerencial para realizar los ajustes pertinentes para el cumplimiento de los objetivos propuestos en los planes vigentes, así como mejorar el diseño de los futuros. Con tal fin, se propone como objetivo diseñar un modelo conceptual y operacional para el seguimiento y evaluación de la gestión territorial, aplicable a los municipios del país, en el marco de los POT y de los requerimientos en materia evaluativa establecidos por la Ley 388 de 1997.

El interés de esta investigación surgió de la experiencia de los autores en la formulación y evaluación de los POT a lo largo de los últimos diez años en varios territorios del país, proyectos en los que se identificaron las necesidades y se 
perfilaron las primeras herramientas evaluativas para dichos planes en diferentes municipios. Esta experiencia permite plantear la posibilidad de construir un modelo integral de seguimiento y evaluación que puede ser utilizado en cualquier municipio colombiano, apoyado en que los procesos de planificación y formulación de los territorios del país han sido dirigidos y orientados por leyes y normativas nacionales que unifican estos procesos, y además porque las administraciones municipales, a pesar de su diferencia en tamaño y complejidad, presentan similitudes en sus características, funciones y responsabilidades en cuanto al ordenamiento territorial.

La evaluación del ordenamiento territorial no solamente involucra las ideas, políticas, objetivos y actividades que se plasmaron en tales planes en un momento dado, sino que debe tener en cuenta, además, todos los procesos y acciones que deberían realizar las diferentes entidades territoriales o municipales para llevar a cabo dichos planteamientos, así como sus capacidades técnicas, tecnológicas y operativas.

La mayoría de los municipios colombianos desconoce la efectividad o el fracaso de las políticas, objetivos y proyectos propuestos para el ordenamiento en sus territorios, desde la perspectiva de los POT. Se establece que lo expuesto anteriormente es el problema principal al que le apunta resolver este artículo, mediante la implementación de instrumentos efectivos de evaluación y seguimiento, ya que no existe una cultura de evaluación en los procesos de construcción del territorio y la ciudad. A partir de los anteriores cuestionamientos surge la pregunta que orientó la investigación: ¿qué elementos teóricos y operacionales debe contener un modelo de evaluación de los Planes de Ordenamiento Territorial que sea aplicable a los municipios colombianos en el marco de la Ley 388 de 1997 y sus normas complementarias?

\section{Metodología}

El desarrollo del modelo se incluye dentro del tipo de investigación aplicada, que busca resolver un problema práctico, en este caso relacionado con la gestión pública, particularmente la gestión del territorio.

El Ministerio de Ambiente, Vivienda y Desarrollo Territorial de Colombia construyó, hace unos años, unas directrices generales para orientar a los diferentes municipios en la evaluación de sus POT, teniendo en cuenta que la gran mayoría debía comenzar a realizar los ajustes y actualizaciones correspondientes a dichos planes formulados hace un poco más de diez años, a raíz de la Ley 388 de 1997. El Ministerio elaboró unas pautas metodológicas básicas con las cuales algunos municipios del país comenzaron a construir unas primeras herramientas evaluativas de sus POT, denominados la mayoría como "Expedientes municipales".

Se utiliza un método inductivo de investigación a partir de experiencias particulares de los investigadores en municipios del Valle del Cauca (Tuluá, Candelaria) así como en el Distrito Capital de Bogotá. En estos casos se hicieron intentos iniciales por evaluar sus respectivos Planes de Ordenamiento Territorial (POT), para retroalimentar los procesos de planificación territorial y realizar los ajustes necesarios. 
El ciclo metodológico utilizado sigue los lineamientos de un modelo tipo RDDA (research, development, dissemination and adoption) (Robson 1993). A partir de los modelos teóricos evaluativos y de gestión seleccionados, así como del estudio y análisis de las experiencias de terreno del grupo de investigación Hábitat y Desarrollo sostenible de la Universidad del Valle, se diseña el modelo conceptual general para la evaluación y el seguimiento a la gestión territorial, el cual entra en etapa de diseminación para su posterior adopción.

Debido a que los procesos de planificación y formulación de los territorios del país han sido dirigidos y orientados por leyes y normativas nacionales (que pretenden incluso unificar los mismos procesos evaluativos), es posible llegar a un modelo general de evaluación de los POT que facilite los procesos de seguimiento y evaluación, y apoye decisiones y ajustes pertinentes.

\subsection{Evaluación y seguimiento en el contexto de la evaluación de programas}

La evaluación, en su definición más amplia, es un tipo de investigación aplicada que intenta determinar el valor de un esfuerzo, de una innovación, una intervención, algún servicio o enfoque determinado (Robson 1993). Según Solarte (2004), una primera tendencia la concibe como un mecanismo para determinar los resultados de una intervención con el objeto de tomar decisiones de carácter gerencial, mientras que una segunda la considera como un proceso constructivista inherente a la acción social, que permite reflexionar y construir colectivamente propuestas para el mejoramiento de la sociedad; esta última le da a la evaluación un alcance más amplio y deliberativo que cuestiona la sociedad, el Estado y la ideología que lo sustenta.

Para Tyler (Stufflebeam y Shinkfield 1985) uno de los primeros autores que propuso una metodología sistemática en el campo educativo, la evaluación se basa en la comparación entre objetivos propuestos y resultados obtenidos, para lo cual se requiere establecer metas generales y objetivos conductuales que deben ser comparados con los resultados, utilizando diseños experimentales y cuasi experimentales, pruebas estandarizadas de medida y el informe clásico de investigación sobre la conveniencia de mantener o modificar los programas (Martínez 1998). A partir del modelo de Tyler, centrado en los objetivos, la evaluación se ha desarrollado evolucionando entre diferentes posturas teóricas y metodológicas, con debates entre lo cuantitativo y lo cualitativo, caracterizándose en la literatura por la presencia de cuatro generaciones de pensamiento, las cuales se relacionan con paradigmas que van desde el pos-positivismo hasta el constructivismo. En los últimos años ha cobrado fuerza la aparición del paradigma pragmático (Patton 2002), así como la aceptación creciente de métodos mixtos (Green y Caracelli 1997).

Stufflebeam y Shinkfield (1985) consideran la evaluación como el proceso de identificar el valor o mérito de algo y proponen metodologías sistémicas que analizan la intervención a través de su contexto, entradas, procesos y productos, con el fin de tomar decisiones que mejoren el programa.

Por su parte, Guba y Lincoln (1989) plantean que la evaluación, en un contexto o situación particular, implica una relación continua de valoración e 
interpretación. Para esta escuela evaluativa, las metodologías apropiadas son de carácter naturalístico e interpretativas, bajo una epistemología constructivista.

La evaluación es considerada hoy un elemento primordial dentro de la denominada Nueva Gerencia Pública (NGP) que busca introducir lógicas de administración privada en el sector público y concentrarse en el logro de resultados (Olías de Lima 2001).

Según la OECD, la evaluación puede cubrir diferentes aspectos tales como pertinencia, logro de objetivos, eficiencia, eficacia, impacto y sostenibilidad. La evaluación determina el valor del programa o política y la información que provee debe permitir tomar decisiones (WP-EV 2010) tanto para beneficiarios como financiadores.

\subsection{La evaluación en el contexto de la gestión y el desarrollo territorial}

La evaluación es una disciplina que, en el campo político y social, facilita el aprendizaje de la sociedad y el control de los ciudadanos hacia sus gobernantes (House 1994; Ospina 2001). Pedir cuentas a estos requiere conocimiento e información, lo cual se consigue mediante procesos evaluativos que develen ante la opinión pública y la ciudadanía lo que el gobierno hace y los resultados de sus políticas. El tema de la evaluación de proyectos y programas públicos no es un tema nuevo y la preocupación actual por la evaluación de la gestión pública se enmarca incluso dentro de una agenda mundial de reforma al Estado y modernización de las instituciones públicas. Puede hablarse además de un cambio de paradigma en la administración pública de los últimos años cuyas manifestaciones concretas varían de país a país, pero cuyas bases filosóficas y conceptuales tienen visos comunes (Ospina 2001).

En los enfoques modernos de gerencia pública, la evaluación es pieza fundamental para reformar al Estado, hacer seguimiento de su desempeño, evaluar los resultados, medir las expectativas y el nivel de satisfacción del ciudadano, y exigir rendición de cuentas al gobernante o funcionario público (Mayne y ZapicoGoñi 1999; Solarte 2004).

Según la CEPAL (1998), en un esfuerzo por modernizar la gestión pública se generaron diversas iniciativas para enfrentar la ineficiencia de la organización burocrática, con dos líneas teóricas: el neoinstitucionalismo y el gerencialismo. La primera hace énfasis en la relación entre políticos y burócratas, mientras que la segunda busca incorporar elementos de la administración privada en la gerencia pública, particularmente conceptos como calidad, eficiencia, incentivos, valor y flexibilidad.

El paso de la gestión pública burocrática a la gestión pública de carácter gerencial (gerencialismo) (Nueva Gestión Pública) ha generado las siguientes tendencias en la práctica de la gestión:

- Necesidad de clarificar y definir las expectativas con relación al desempeño de las organizaciones y las políticas y programas que sus gerentes administran. 
- Necesidad de dar mayor flexibilidad y autonomía al gerente público pero, a su vez, solicitando mayor responsabilidad y un claro rendimiento de cuentas frente al desempeño de su organización.

- Reducción del tamaño del Estado y su participación directa en la producción de servicios, reemplazando esta por mecanismos como la privatización y contratos con otros proveedores.

- Tratamiento de las agencias públicas como repositarias de "contratos" o "acuerdos" con los ministerios u otras jurisdicciones para las que funcionan (Ospina 2001).

Según la CEPAL (1998), la modernización se concentra en la aplicación de principios, técnicas y prácticas de gestión para aumentar el impacto y la eficiencia de los programas y proyectos. Los principios utilizados son: equidad, focalización, impacto, participación ciudadana, articulación, flexibilidad, entre otros. En el campo de la gestión pública, los programas y proyectos sociales juegan un papel indispensable como materialización u operacionalidad de las políticas públicas (CEPAL 1998).

\section{Modelo conceptual para la evaluación y el seguimiento a los POT}

El modelo está basado en el enfoque de evaluación de cadena de resultados (Vedung 1997; CEPAL 1998) en donde se determinan los productos, efectos e impactos del POT, el cual se integra con un análisis de la gestión institucional y el diseño del plan. La evaluación utiliza un enfoque mixto (Greene y Caracelli 1997) que integra métodos cuantitativos y cualitativos, cada uno orientado a información específica para la cual es pertinente, por ejemplo, elementos cuantitativos para medir diferentes temas del territorio y cualitativos para valorar la percepción de los diferentes actores.

Así mismo, la evaluación a realizar a partir de la formulación de los POT será concomitante (es decir, que pretende analizar los resultados en cualquier momento durante la ejecución o realización de los POT), teniendo en cuenta que la evaluación territorial es un proceso permanente, continuo, de indagación y valoración de la planificación y gestión territorial, de la ejecución y la finalización de los planes, programas y proyectos del proceso de construcción territorial (INAP 1997). El modelo plantea la realización de la evaluación y el seguimiento ex post, después de iniciada la fase de operación del Plan y de sus programas o proyectos, analizando principalmente la población objetivo (impacto), producción del plan, sus programas o proyectos (eficiencia y eficacia) y su capacidad de gestión (efectividad).

Se definen cuatro niveles evaluativos: Nivel I: Suficiencia y articulación; Nivel II: Productos y gestión de proyectos; Nivel III: Gestión administrativa; y Nivel IV: Impactos y efectos. Estos cuatro niveles se complementan con un análisis de articulación y suficiencia que plantea la metodología del Ministerio de Ambiente, Vivienda y Desarrollo Territorial (2007), lo cual permite una evaluación más integral de la gestión, partiendo del análisis de marco lógico donde se ligan actividades, resultados, propósitos y objetivos de un programa y sus proyectos, 
políticas, indicadores necesarios, fuentes de información e hipótesis en los planteamientos establecidos en los POT. Estas dimensiones se aplican a una serie de variables claves que constituyen la planificación del territorio y reflejan el alcance de la gestión realizada en múltiples dimensiones.

\subsection{Ciclo del seguimiento y la evaluación en los procesos de planificación territorial de los POT}

Tanto el seguimiento como la evaluación en la planificación territorial son un proceso continuo y sistemático, que debe implementarse de manera paralela con la puesta en marcha del POT, como la misma Ley 388 de 1998 lo plantea. El seguimiento para el caso de los POT podría definirse como una función continua cuyo principal objetivo es proporcionar a las administraciones y a los principales actores o interesados, en este caso en la planificación territorial, indicaciones tempranas de progreso, o de la falta del mismo, en el logro de resultados.

La evaluación en la gestión territorial es un ejercicio selectivo que intenta determinar, de manera sistemática y objetiva, los progresos del POT hacia un efecto o impacto determinado. La evaluación no es un proceso aislado, sino un ejercicio que se integra a la formulación y ejecución del plan para identificar ajustes que permitan retroalimentar la formulación. Este ciclo plantea implícitamente una profunda relación entre planificación y evaluación en la medida en que se alimentan mutuamente y constituyen la base del aprendizaje institucional.

Por tanto, es importante que el modelo que se plantee propenda no solo por determinar una evaluación por objetivos predeterminados (modelo clásico) sino que, además, permita analizar el grado de avance de unos efectos e impactos deseables, mediante el seguimiento y la evaluación de varios niveles conceptuales que involucran diferentes dimensiones del ordenamiento y la gestión territorial produciendo, asimismo, información útil (que retroalimente) a los actores involucrados en estos procesos.

\subsection{Elementos claves para la construcción del modelo}

Es preciso analizar la pertinencia de un modelo para la evaluación y el seguimiento a la gestión del ordenamiento de los territorios, a partir de los planteamientos estipulados en los POT, teniendo en cuenta, además, que dichos planes fueron construidos todos bajo las directrices y políticas generales de la Ley 388 y demás normas complementarias. Es decir, el planteamiento de un modelo podría establecer niveles conceptuales comunes a todos los territorios del país, pero con énfasis diferentes, de acuerdo a las necesidades y situaciones particulares de cada región.

Es pertinente establecer elementos de diseño a partir de los conceptos y el estudio del arte de la evaluación en el contexto de la evaluación de proyectos y programas sociales, así como de las políticas de planificación territorial establecidas en la actualidad en Colombia. 


\subsubsection{Perspectivas de análisis}

La planificación territorial en nuestro país se puede explicar desde dos perspectivas: la territorial (municipio, departamento, comunidad) o la institucional (gobierno, entidad, dependencia). Desde la perspectiva territorial se analiza el ámbito geo-espacial y aquellas actividades que potencian el desarrollo de los aspectos físicos, económicos, sociales, demográficos y culturales, incluyendo los POT, su diseño y ejecución (Solarte 1999).

La perspectiva institucional analiza la planificación a nivel de las entidades administrativas, centralizadas o descentralizadas, que conforman el gobierno de la entidad territorial. Se incluyen los diferentes planes de las dependencias del estado, desde lo estratégico hasta lo operacional.

Mediante la planificación territorial se establece el curso de acción de manera concertada entre la ciudadanía y el gobierno, el cual es operacionalizado a través de los planes de las diferentes instituciones que tratan los problemas identificados, buscando soluciones y orientado las acciones a nivel institucional. Según Solarte (1999):

\footnotetext{
Los planes de desarrollo generalmente son elaborados por las administraciones municipales teniendo como insumos básicos los diagnósticos realizados por los equipos técnicos, el programa de gobierno inscrito, la consulta ciudadana y, en algunos casos, los planes de desarrollo local realizados por las comunidades. Sobre esta base se establecen las prioridades que, dada la limitación en la duración del período de gobierno, no garantizan que se consideren estrategias de largo alcance $(1999,342)$.
}

Los POT alcanzan un horizonte un poco más amplio, pues generalmente son concebidos para realizarlos en por lo menos tres administraciones de gobierno, lo que permite manejar, en cierto modo, mejor el tema de estrategias a largo plazo. Sin embargo, en muchos casos se duplican aspectos que son tenidos en cuenta también en los planes de desarrollo social y económico, lo cual plantea adicionalmente un problema práctico, por cuanto la planificación del desarrollo económico y social no puede concebirse independientemente y con un horizonte de planificación inferior al del ordenamiento territorial (Solarte 1999).

\subsubsection{Utilización de enfoques cuantitativos}

Es pertinente la utilización de enfoques de evaluación cuantitativos, que permitan analizar y hacer seguimiento a los diferentes temas del territorio mediante información que pueda ser medible para sustentar las decisiones a tomar de una manera más objetiva, sin desconocer, claro está, la posibilidad de incluir alguna información cualitativa. Teniendo en cuenta lo expresado por Bustelo (1999), se pretende que el modelo permita que "las decisiones de los actores no se basen en meras opiniones sino en datos y estudios sistemáticos sobre los procesos, los productos y el impacto de la acción de los poderes públicos" $(1999,3)$.

Según el INAP (1997), la medición del desempeño incluye básicamente los productos medibles que se generan a partir de los recursos involucrados, los resultados en relación con los objetivos propuestos y, el impacto en la sociedad y el medio ambiente. 


\subsubsection{Niveles evaluativos}

Se deben establecer los diferentes niveles en los que se centrarán los procesos de evaluación, que para este caso se basan en las variables que plantea el modelo de la Comisión Económica, tales como la población objetivo, el impacto, la producción y la capacidad de gestión, e incluir dentro de estos niveles el análisis de los planteamientos del POT en cuanto a su coherencia, articulación y suficiencia, etc.

\subsection{4 Áreas o dimensiones de impacto}

Teniendo en cuenta el anterior punto, se deben establecer áreas de impacto en los diferentes componentes del Plan para, posteriormente, realizar su respectiva evaluación. Es conveniente que sea un ente o una unidad externa la que evalúe el impacto global de las acciones de la administración. Por su parte, las diferentes entidades de la administración, deben hacer el seguimiento y evaluación de las acciones en los niveles de utilización de recursos, implantación de procesos y obtención de resultados de corto plazo. Cada unidad o entidad de la administración municipal debe ser responsable de su propia planificación, en función de contribuir a la obtención de los objetivos plasmados en el plan de desarrollo y en el POT. La evaluación de la gestión en las distintas entidades debe enfocarse en establecer los logros obtenidos en esta dirección. De la misma manera, es importante que en los procesos de planificación y evaluación se involucren activamente los diferentes actores, incluida la misma comunidad.

\subsubsection{Interrogantes a resolver}

El modelo de evaluación y seguimiento debe responder, en primer lugar, a aquellos interrogantes básicos que han sido establecidos por la guía del Ministerio de Ambiente, Vivienda y Desarrollo Territorial (2007) en cuanto a: suficiencia; articulación; cumplimiento de metas u objetivos; desarrollo del modelo territorial o físico (impacto); y ejecución de proyectos, y para tal efecto deben establecerse indicadores apropiados. La respuesta a estos interrogantes permitirá el análisis evaluativo que, a su vez, retroalimentará los esfuerzos de planificación.

De otra parte, es importante analizar la coherencia entre políticas, objetivos, metas y actividades del modelo de marco lógico (Martínez y Fernández 2012) y la importancia del impacto, como eje primordial para el desarrollo de los modelos de gestión y organización.

\subsection{Modelo organizativo para las administraciones municipales}

Es necesario replantear el modelo organizativo de las administraciones municipales, con el fin de hacerlo más acorde a las necesidades de planificación y evaluación territorial (planteamiento reiterativo de la CEPAL) cuya función objetivo debería ser maximizar el impacto externo y la eficiencia interna de la siguiente forma: Impacto /Productos /Procesos /Actividades/ Modelo de Gestión/ Modelo de Organización (CEPAL 1998). Igualmente, para asumir estos nuevos enfoques de trabajo, tanto para la planificación como para un adecuado sistema 
de evaluación y seguimiento del territorio, es necesario conformar estructuras con conocimientos y habilidades en temas como: enfoque al trabajo multidisciplinario en los distintos temas del desarrollo y el ordenamiento, manejo de metodologías modernas de planificación y evaluación, manejo de metodologías de planificación territorial en sus diferentes componentes y manejo de nuevas tecnologías, tales como los Sistemas de Información Geográfica, análisis estadístico, software especializado, entre otros, para la gestión de proyectos y la toma de decisiones. Conocimientos de legislación territorial y del desarrollo, Gerencia de proyectos y participación ciudadana y metodologías de socialización y/o participación (Solarte 1999).

Ante todo, el modelo de evaluación y seguimiento debe ser operativo e informar oportunamente sobre el estado de la gestión territorial, sin convertirse en un sistema complejo que requiera una dedicación mayor que los mismos procesos de planificación. Por tanto, debe plantear un mínimo de indicadores claves, que permitan ser medidos constantemente sin necesidad de grandes inversiones. Igualmente, debe establecer un modelo de organización institucional para su correcto funcionamiento, que permita que los indicadores sean constantemente actualizados como parte de los procesos mismos de gestión de cada una de las entidades a cargo de las temáticas en los territorios.

Para la construcción del modelo es necesario que el municipio cuente con un Sistema de Información Geográfica (SIG, o GIS en inglés) actualizado, que le permita vincular información espacial, y no espacial y facilite el análisis y seguimiento a la gestión territorial. Dicho sistema debe poder informar, con una debida oportunidad en el tiempo, sobre los diferentes fenómenos o avances en la implementación de los diferentes proyectos o actividades del Plan de Ordenamiento Territorial, con información veraz y bajo parámetros de calidad. En la actualidad, los SIG son una tecnología muy importante dadas las posibilidades que ofrecen para vincular información espacial (planos) y no espacial (bases de datos), que contribuyen de manera importante para la toma de decisiones en temas tan complejos como la planificación territorial. Para manejar o analizar la información es necesario definir, como primera medida, los objetivos o requerimientos de los usuarios, resumidos en las entidades, variables o coberturas que se necesitan o que se utilizan dentro del análisis, sus atributos y sus interrelaciones; clasificarlos, codificarlos y asignar la norma de representación.

Para el caso específico de la evaluación y el seguimiento a la gestión territorial y, en general, al ordenamiento de los territorios, la misma Ley 388 de 1997 estableció la necesidad de contar con un adecuado sistema de información que sustente los diagnósticos y la definición de políticas, así como la formulación de planes, programas y proyectos de ordenamiento, al cual denominó "Expediente Municipal" (antes Expediente Urbano). La definición que hace la Ley 388 de los Expedientes Municipales alude principalmente a un Sistema de Información; es decir, se entiende que para que se pueda evaluar o para que se pueda construir un sistema de evaluación territorial se debe contar con un sistema de información completo, preciso y veraz, pues este sistema es la base sobre la cual se apoyará cualquier instrumento de seguimiento y evaluación al ordenamiento.

Un adecuado sistema de información territorial municipal debe contar con herramientas y tecnología de vanguardia que permitan relacionar datos gráficos 
(cartografía digital) con bases de datos alfanuméricos, para así facilitar las labores de evaluación y seguimiento a los POT, e igualmente de soporte técnico a las decisiones que en materia de planeamiento o generación de políticas territorial se tomen para el presente o hacia el futuro. De esta manera, el SIG que se diseña para la evaluación y el seguimiento al ordenamiento y a la gestión territorial debe tener, como primera finalidad, la de facilitar las labores de monitoreo y de toma de decisiones en lo relacionado con el territorio.

Igualmente, la evaluación requiere encontrar métodos o estrategias para la captura de información rápida y precisa que le permita ser más operativa y acertada; por tanto, el desarrollo de sistemas de información paralelos a la evaluación son de vital importancia. Según Chelimsky (1998), la adquisición de información es una de las mayores dificultades existentes a la hora de evaluar, sobre todo cuando se requiere evaluar el resultado y el impacto de programas y actividades. Por lo anterior, el modelo propuesto consta de dos partes: primero, niveles conceptuales para la evaluación y el seguimiento; segundo, dimensiones estructurantes a evaluar del ordenamiento territorial.

\subsubsection{Niveles conceptuales para la evaluación y el seguimiento a la gestión territorial}

El modelo propuesto pretende evaluar y hacer seguimiento a la gestión territorial a partir de los planteamientos de los POT de las administraciones municipales del país, así como reflejar los aspectos organizativos del Plan relacionados con la forma de departamentalización de las actividades y organización del trabajo. La propuesta plantea la necesidad de evaluar, de forma sistemática y continua, los diferentes componentes de un POT a partir de las variables que plantea la Comisión Económica como son el impacto, la producción o productos, teniendo en cuenta, a su vez, la plataforma administrativa, tecnológica y de gestión, que debe estar en las administraciones municipales.

Para la construcción del modelo se definen, en primera medida, los niveles conceptuales que se evaluarán y bajo los cuales se realizará el monitoreo o seguimiento, teniendo en cuenta las diferentes áreas que maneja la gestión territorial municipal. Para el caso de este modelo, se propone la utilización de niveles basados, en parte, en las variables que plantea la CEPAL, debido a que se considera que dichas variables agrupan las diferentes áreas relacionadas con la gestión territorial en cuanto al impacto, la producción y la capacidad de gestión, y un nivel que consiste en realizar una evaluación de la articulación y suficiencia del POT y un análisis general de los contenidos del plan: si estos son coherentes, si están completos y si hay articulación entre sus partes. A partir de estos niveles se construirán los diferentes indicadores que, mediante matrices, se organizarán y analizarán en el sistema de seguimiento; posteriormente se planteará una metodología a seguir para la implantación o montaje del modelo de evaluación y seguimiento y la forma en que deberá operar el modelo de evaluación dentro de las administraciones municipales. La Figura 1 describe los niveles o fases a evaluar dentro del modelo; como se observa, están representados por cuatro anillos o niveles así: 
Figura 1. Niveles y dimensiones conceptuales para la evaluación

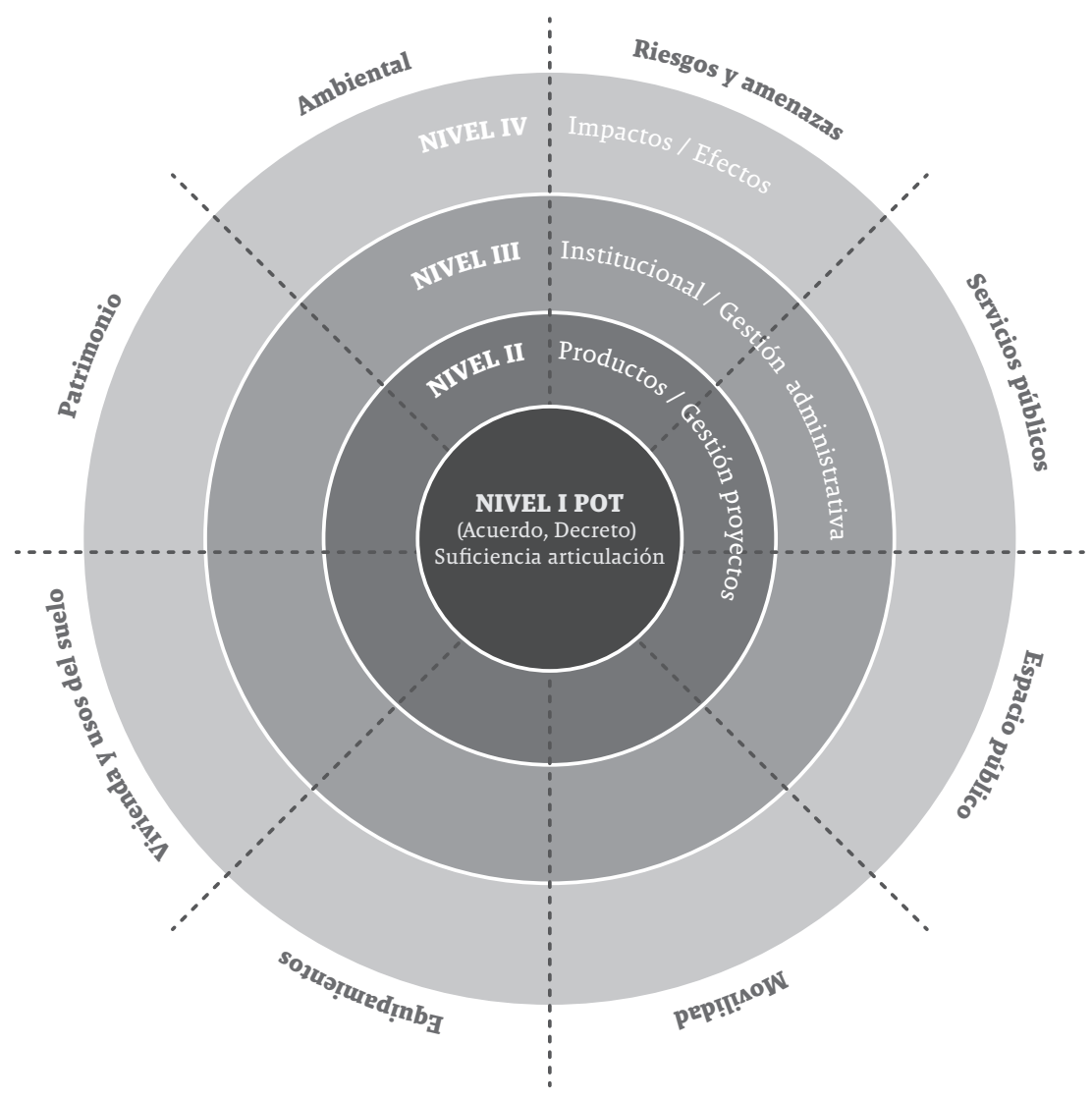

Fuente: elaboración propia.

Un primer nivel relacionado con el análisis de la suficiencia, la articulación y la coherencia de los planteamientos plasmados en los POT; un segundo nivel, donde se evalúan los avances de los proyectos o programas establecidos (gestión de proyectos); un tercer nivel, que evaluará la gestión administrativa y un cuarto nivel que muestra si los fines últimos del desarrollo y la planificación territorial se están dando o no y si los esfuerzos han valido la pena, así como detecta de cierta manera los cambios que deben realizarse en el modelo organizativo de las administraciones y en la gestión territorial, para alcanzar los impactos y efectos deseados. Los anillos en sí representan cada nivel a evaluar, desde el centro (que analiza los planteamientos del POT) hasta el exterior, que analizará los diferentes efectos o impactos que se tuvieron; este último anillo podría calificarse de cierta forma como uno de los más importantes debido a que refleja, en últimas, si los objetivos o metas del ordenamiento territorial se dieron o no y de qué forma. 


\subsubsection{Dimensiones estructurantes a evaluar del ordenamiento territorial}

Las dimensiones o temas estructurantes del ordenamiento territorial aparecen en la periferia de los círculos (Figura 1); están constituidas por aquellos temas que participan en la planificación física de los espacios, tanto urbanos como rurales, donde intervienen múltiples temáticas que son de vital importancia en el desarrollo físico y socioeconómico de los diferentes territorios. Cada uno de los niveles anteriormente descritos se evaluarán para cada uno de los temas o dimensiones del ordenamiento territorial: dichas dimensiones agrupan las temáticas que el ordenamiento de los territorios y la planificación urbana deben considerar para una adecuada utilización del suelo y el mejoramiento de las condiciones de los asentamientos humanos y la calidad de vida de sus habitantes, entre las que se encuentran las siguientes:

\subsubsection{Riesgos y amenazas}

Bajo esta temática se analiza y estudia, por tanto, lo relacionado con las posibles amenazas y riesgos que se presentan en los territorios, especialmente por fenómenos de inundación, remoción en masa (derrumbes), sismicidad, erupciones volcánicas, tsunamis o maremotos, huracanes e incendios, entre otros.

\subsubsection{Servicios públicos}

En el ordenamiento territorial, el tema de los servicios públicos es uno de los factores que condiciona la calidad de vida de los ciudadanos, pues determina circunstancias de salubridad e integridad en sus pobladores, quienes deben tener acceso al agua potable, a la energía o al saneamiento básico (recolección y disposición final de desechos).

\subsubsection{Espacio público}

Para el caso particular del ordenamiento territorial, bajo esta temática se plantea una estructura de espacios públicos que muchas veces involucra áreas o suelos de protección ambiental y que permite establecer unas redes de parques o zonas de recreación pasiva o activa. Se generan políticas y normativas sobre el uso y la intervención en dichos espacios, así como para generar nuevos lugares de esta índole, con el objeto de mejorar las condiciones de calidad ambiental y de vida de las comunidades.

\subsubsection{Movilidad}

El tema de movilidad en el ordenamiento territorial tiene que ver con la eficiencia y eficacia de las infraestructuras y/o sistemas viales y de transporte que inciden directamente en la productividad de los territorios y en las condiciones de vida de sus pobladores. Se analizan bajo esta temática principalmente los sistemas de infraestructuras viales y de movilidad, incluyendo la movilidad de peatones, automóviles, sistemas de transporte masivo, bicicletas, carga y las diferentes alternativas de transporte (terrestre, marítimo, aéreo, etc.). 


\subsubsection{Equipamientos}

El tema de equipamientos públicos en la planificación y ordenamiento de los territorios permite establecer las infraestructuras necesarias que se requieren para que su población cuente con los servicios básicos de salud, educación, cultura, recreación y bienestar social, entre otros. Se deben establecer estándares mínimos y lugares adecuados para que los ciudadanos tengan acceso a estos servicios indispensables para su calidad de vida y su bienestar general.

\subsubsection{Vivienda y usos del suelo}

Los usos del suelo incluyen los diferentes destinos que se le pueden dar al territorio, en sus distintos tipos (rural, urbano, de expansión, etc.). Están incluidos los usos que se pueden dar en los suelos rurales, tales como los agropecuarios o agroindustriales. Analiza, además, el impacto que cada uso del suelo puede tener sobre los sistemas ambientales o ecosistemas, así como en la población que los habita.

Se incluye en esta temática la generación de nuevos territorios para vivienda, uno de los principales usos al interior de las ciudades y centros poblados y en donde es de vital importancia estudiar y analizar la calidad de la construcción de las edificaciones destinadas para este fin (Ley 388 de 1997).

\subsubsection{Patrimonio}

El patrimonio a nivel territorial se refiere a aquellos bienes que una sociedad determina conservar, por su valor cultural, natural o tradicional. El Ministerio de Vivienda y Urbanismo de Chile (s/f) plantea que el patrimonio urbano "comprende las edificaciones y los espacios públicos cuya forma constitutiva es expresión de la memoria colectiva, arraigada y trasmitida, los que en forma individual o en conjunto revelan características culturales, ambientales y sociales que manifiestan y fomentan la cultura y la consolidación social" (Ministerio de Vivienda y Urbanismo de Chile s/f).

\subsubsection{Ambiente y recursos naturales}

En el ordenamiento territorial se considera el suelo como un recurso natural en donde se deben planear y ordenar todos sus usos en forma integral. Esta integración debe hacerse atendiendo, de un lado, todos los factores ambientales, sociales y económicos $\mathrm{y}$, por otro, todos los componentes del medio ambiente y los recursos conjuntamente. La consideración integral facilita opciones y compensaciones adecuadas, llevando a su máximo nivel la productividad y la utilización sostenibles (CEPAL 2001).

\section{Conclusiones}

El presente modelo de evaluación ha sido diseñado sobre los parámetros de planificación territorial y políticas de ordenamiento territorial que han sido implementados hasta el día de hoy en nuestro país, fundamentados en leyes y normativas que, a nivel nacional, homogenizan los contenidos, procesos y 
metodologías para la construcción de los planes de ordenamiento territorial, e igualmente unifican el funcionamiento de las entidades territoriales. Por tanto, puede ser implementado de manera general en cualquiera de los territorios del país que así lo requiera.

A diferencia de las metodologías construidas a la fecha para la evaluación y el seguimiento a los planes de ordenamiento territorial, el modelo propuesto pretende realizar una evaluación integral a la gestión del ordenamiento territorial, organizada por niveles, los cuales abarcan todos los procesos que implica el desarrollo físico de los territorios. Así mismo, de manera transversal, el modelo propuesto evalúa en cada uno de estos niveles las grandes temáticas o dimensiones del ordenamiento y la planificación territorial, temáticas que a su vez, pueden ser evaluadas por separado y de este modo encontrar problemáticas puntuales, áreas a priorizar, etc.

El modelo ha incorporado, a diferencia de las experiencias evaluativas hasta ahora realizadas en el país, la evaluación de la gestión institucional (nivel III) por su importancia en el desarrollo de lo planificado dentro de los POT, pues tiene que ver con las capacidades operativas, técnicas y tecnologías que poseen las administraciones territoriales para llevar a cabo lo planteado, así como las capacidades financieras para llevar a buen término el desarrollo de los programas o proyectos. Por otro lado, en el nivel IV se propone una serie de indicadores claves para la gestión y la evaluación del ordenamiento territorial, teniendo en cuenta su importancia a la hora de medir el impacto y el estado de una temática particular, sin que esto conlleve a su única determinación, pues a medida que un municipio cuente con información más completa puede realizar mejores análisis y manejar igualmente un grupo específico de indicadores de acuerdo con sus prioridades o necesidades más importantes.

Uno de los principales insumos en un sistema de evaluación y seguimiento es, sin duda alguna, la información. Para el caso específico de la evaluación del territorio, la información geográfica es la base fundamental de los procesos de evaluación y gestión de los territorios, la cual, además de necesitarse lo más actualizada y precisa posible, debe tenerse de manera oportuna.

El principal obstáculo de los sistemas de evaluación y de los observatorios territoriales que se han tratado de implementar en nuestro país, es precisamente el no contar con un Sistema de Información Geográfica oficial y fiable, o el tener información dispersa entre todas las instituciones que componen una administración territorial. Dichos sistemas (SIG) apenas han comenzado a ser construidos e implementados en algunos municipios pues, además de ser necesaria una inversión importante para su construcción y mantenimiento, se debe contar con personas especializadas en el manejo y construcción de datos e información. Por ello, es importante recalcar que hasta tanto un municipio no cuente por lo menos con un Sistema de Información Geográfica oficial de su territorio, muy difícilmente podrá implementar un sistema de evaluación y seguimiento al ordenamiento territorial que sea realmente operativo.

En el modelo se plantea la institucionalización de un sistema de evaluación y seguimiento y se propone que las oficinas de planeación sean las que lideren los procesos de planificación y generación de políticas territoriales. De la misma manera, se propone una metodología acorde a las actuales estructuras internas 
de las administraciones, para lograr la viabilidad de su implementación y su operatividad a lo largo de los años. Sin embargo, como en la mayoría de las actividades o procesos de las entidades públicas, es necesario contar con un verdadero compromiso político de parte de las instituciones y de los gobernantes municipales para que se garanticen los procesos de evaluación y seguimiento a corto, mediano y largo plazo y lograr, de este modo, los objetivos esperados de esta herramienta, como son facilitar los procesos de toma de decisiones y la retroalimentación en los procesos de planificación territorial.

La metodología propuesta para la implantación del modelo en cualquier territorio del país pretende facilitar los procesos, coordinando las funciones y responsabilidades de las diferentes dependencias que conforman una administración municipal, que a la vez permita aunar esfuerzos y recursos en pro de construir y mantener un sistema evaluativo como herramienta fundamental para la retroalimentación, y la toma de decisiones en la planificación territorial. Es importante recalcar que estos sistemas deben propender por la dinámica de sus procesos, que facilite las actividades de evaluación sin que se conviertan en un esfuerzo más grande que el mismo proceso de planificación.

\section{Referencias bibliográficas}

Bustelo, María. Diferencias entre la evaluación e investigación: una distinción necesaria para la identidad de evaluación de programas. Madrid: Universidad Complutense de Madrid-España, 1999. [En línea]. http://www.unidaddeigualdad.es/documentos_contenidos/1150_69457135_DiferenciasInvestigEval.pdf (último acceso: 24 de noviembre de 2014).

Chelimsky, Eleanor. «The Role of Experience in Formulating Theories of Evaluation Practice». American Journal of Evaluation, Vol. 19, $\mathrm{n}^{\circ}$ 1, 1998: 35-55.

Comisión Económica para América Latina y el Caribe, CEPAL. Gestión de programas sociales en América Latina, Serie 25, Políticas sociales, Vol. I. Santiago de Chile: Naciones Unidas, 1998.

- El ordenamiento territorial como opción de políticas urbanas y regionales en América Latina y el Caribe [Versión electrónica], (Serie 45 Medio ambiente y desarrollo). Santiago de Chile: Naciones Unidas, 2001.

Greene, Jennifer, y Valerie Caracelli. «Defining and Describing the Paradigm Issue in Mixed - Method Evaluation». En Advances in Mixed-Method Evaluation: The Challenges and Benefits of Integrating Diverse Paradigms. Serie: New Directions for Evaluation. Number 74. San Francisco, CA.: Bass Publishers, American Evaluation Association, 1997.

Guba, Egon, e Yvonna Lincoln. Fourth Generation Evaluation. Newbury Park, California: SAGE Publications, 1989.

House, Eduard. Evaluación, ética y poder. Madrid: Ediciones Morata, 1994.

Instituto Nacional de la Administración Pública-INAP. Evaluación en la Gestión Pública, conceptualización, modalidades y nuevas perspectivas (serie: documento de apoyo a la capacitación) [versión electrónica]. Buenos Aires: INAP, 1997.

Martínez, Carlos. «La teoría de la evaluación de programas». Educación XXI, Vol. $1, n^{\circ} 1,1998: 73-91$. 
Martínez, Rodrigo, y Andrés Fernández. Metodologías e instrumentos para la formulación, evaluación y monitoreo de programas sociales. S/c: CEPAL, 2012.

Mayne, Jane, y Eduardo Zapico-Goñi, «Effective Performance Monitoring: A Necessary Condition for Public Sector Reform». En: Monitoring Performance in the Public Sector, editado por Jane Mayne y Eduardo Zapico-Goñi, 11-17. New Brunswick: Transaction Publishers, 1999.

Ministerio de Ambiente, Vivienda y Desarrollo Territorial. Guía metodológica para la conformación y puesta en marcha del expediente municipal [versión electrónica]. Bogotá: Ministerio de Ambiente, Vivienda y Desarrollo Territorial, 2007.

Ministerio de Vivienda y Urbanismo. «Patrimonio urbano». En Ministerio de Vivienda y Urbanismo, Gobierno de Chile, (s/f). [En línea]. http://www.minvu.cl/ opensite_20061113163034.aspx (último acceso: 15 de noviembre de 2014).

Ospina, Sonia. «Evaluación de la gestión pública: conceptos y aplicaciones en el caso latinoamericano». Revista del CLAD Reforma y Democracia, n 19, 2001. [En línea]. http://old.clad.org/portal/publicaciones-del-clad/revista-clad-reforma-democracia/articulos/ol9-febrero-2001-1/evaluacion-de-la-gestion-publicaconceptos-y-aplicaciones-en-el-caso-latinoamericano-1 (último acceso: 24 de noviembre de 2014).

Patton, Mattew. Qualitative Evaluation \& Research Methods (за. ed.). Thousand Oaks: Sage Publications, 2002.

Robson, Carl. Real World Research. Londres: Blackwell, 1993.

Solarte, Leonardo. «Los sistemas de planeación y evaluación municipal en el contexto de la descentralización». En La reestructuración de los gobiernos locales: la experiencia de Armenia, compilado por Edgar Varela, 101-109. Santiago de Cali: Universidad del Valle, 1999.

Las evaluaciones de políticas públicas en el Estado liberal. Santiago de Cali: Universidad del Valle, 2004.

Stufflebeam, Daniel, y Anthony Shinkfield. Evaluación sistemática: guía teórica y práctica. España: Paidós Ibérica S.A., 1985.

Vedung, Evert. Public Policy and Program Evaluation. New Brunswick (USA): Transaction Publishers, 1997.

WP-EV. Glosario de los principales términos sobre evaluación y gestión basada en resultados. Francia: OECD PUBLICATIONS, 2010. [En línea]. http://www.oecd. org/development/evaluation/2754804.pdf (último acceso: 24 de noviembre de 2014).

\section{Normatividad}

Congreso Nacional de la República de Colombia. Ley 388 de 1997 de ordenamiento territorial. Bogotá: Congreso Nacional de la República de Colombia, 1997. 